\title{
Morphogenesis is coordinated with nuclear division in germinating Aspergillus nidulans conidiospores
}

\author{
Steven D. Harris \\ Tel: +1 860679 2774. Fax: +1 860679 1239.e-mail: sharris@nso2.uchc.edu
}

Department of

Microbiology, University of Connecticut Health Center, Farmington, CT 06030-3205, USA

\begin{abstract}
Germinating Aspergillus nidulans conidiospores switch to polarized apical growth following an initial period of isotropic expansion. At the same time, they re-enter the nuclear division cycle. The relationship between spore polarization and nuclear division was investigated by testing the effect of cell cycle inhibitors and temperature-sensitive cell cycle mutations on spore morphogenesis. On rich media, it was found that spore polarization is delayed if completion of the first mitosis is blocked. The observed delay may be dependent upon the activity of the mitosis-promoting NIMA kinase. An additional mechanism appears to prevent polarization as the spore progresses through its first S phase. In contrast, on poor media, spore polarization does not require completion of the first mitosis. These observations suggest that spore morphogenesis is influenced by cell cycle signals in a growth-dependent manner.
\end{abstract}

Keywords: morphogenesis, polarity, cell cycle, spore germination

\section{INTRODUCTION}

One of the distinguishing features of fungal cells is their highly polarized mode of growth. Both yeast cells and hyphal cells grow by cell surface expansion at specified cortical sites. The transport of vesicles laden with new membrane and cell wall material to these sites is facilitated by polarization of the cytoskeletal network (for a review, see Gow, 1995). Although the same general mechanisms are likely to be involved in controlling the establishment of hyphal polarity in budding yeast and filamentous fungi (for a review, see Kron \& Gow, 1995), it is important to note that hyphal cells are organized in a fundamentally different manner to yeast cells (Harris, 1997). In particular, hyphal cells maintain a fixed axis of polarized growth, whereas yeast cells undergo a transient period of depolarization (i.e. isotropic growth) coincident with mitosis and cell division. Furthermore, during cell division, hyphal cells organize an actin ring at the division site while simultaneously retaining an actin cap at the tip (Harris et al., 1994). These differences suggest that the mechanisms regulating cellular morphogenesis in hyphal cells and yeast cells must differ at some level.

Abbreviations: CMI, chromosome mitotic index; HU, hydroxyurea; ts, temperature-sensitive.
Cellular homeostasis depends upon the existence of regulatory mechanisms that ensure that morphogenesis is integrated with growth and nuclear division. In budding yeast, these processes are coordinated with each other during a step in the cell cycle termed START (Pringle \& Hartwell, 1981). The nutritional status of the cell is thought to be assessed by the protein kinase A (PKA) pathway, which in turn modulates the temporal expression of the G1 cyclins (Baroni et al., 1994; Tokiwa et al., 1994). Cyclin-dependent kinase (cdk) activity associated with the G1 cyclins subsequently directs a number of events, including entry into $S$ phase (Dirick et al., 1995), polarization of the actin cytoskeleton (Lew \& Reed, 1993) and activation of a mitogen-activated protein (MAP) kinase pathway dedicated to promoting cell wall biosynthesis (Madden et al., 1997). The switch from apical to isotropic growth that occurs upon entry into G2 is triggered by cdk activity associated with the mitotic cyclins (Lew \& Reed, 1993). In contrast to budding yeast, very little is known about the mechanisms which coordinate morphogenesis with growth and nuclear division in filamentous fungi. One pathway that could potentially serve this function is the PKA pathway. In particular, several studies have shown that PKA-mediated signalling plays a pivotal role in controlling polarized growth in many filamentous fungi (Gold et al., 1994; Bruno et al., 1996; Kronstad, 1998). 
However, it is not yet known if this pathway acts to regulate growth in hyphal cells as it does in yeast.

The filamentous fungus Aspergillus nidulans forms asexual spores (conidia) which contain a single nucleus arrested in G1 (Bergen \& Morris, 1983). During spore germination, conidia undergo an initial period of isotropic expansion before switching to polarized growth and forming an elongating germ tube. Concomitant with these morphogenetic events, spores resume general metabolic activity and re-enter the nuclear division cycle (d'Enfert, 1997). The mechanisms regulating the process of spore germination in A. nidulans are just beginning to be understood. It has been shown that the constitutively activated form of Ras prevents the switch from isotropic to polarized growth, which led to the proposal that down-regulation of Ras activity is required for spore polarization (Som \& Kolaparthi, 1994). Although this result suggests that Ras signalling plays a direct role in the control of morphogenesis, it remains possible that Ras may be involved in growth control and the establishment of a cell size threshold for polarization. In addition, the relationship between spore polarization and nuclear division has not been carefully examined in A. nidulans. Although conidia have been observed to undergo nuclear division prior to spore polarization, it is not known if the switch to polarized growth is dependent upon the completion of a specific cell cycle event.

Nuclear division in A. nidulans is controlled by the activity of two distinct protein kinases (Osmani \& Ye, 1996). The cdk encoded by nim $X, \mathrm{p} 34^{\text {nim } X}$, is required for passage through START and for entry into mitosis (Osmani et al., 1994). Activation of $\mathrm{p} 34^{\text {nimX }}$ requires association with the cyclin $\mathrm{p} 56^{\text {nime }}$ (O'Connell et al., 1992). Furthermore, during G2, p34 $4^{\text {nimX }}$ is converted into an active form by the tyrosine phosphatase $\mathrm{p} 80^{\text {nim T }}$ (O'Connell et al., 1992). In addition to p34 ${ }^{\text {nimX }}$, mitotic entry in A. nidulans requires the activation of the NIMA protein kinase. NIMA activity gradually accumulates as hyphal cells progress through G2 and reaches a maximum in early mitosis (Osmani et al., 1988). Attainment of maximal activity requires hyperphosphorylation of NIMA by p34 $4^{\text {nimX }}$ at the G2/M boundary (Ye et al., 1995). Mitotic progression is dependent upon the subsequent destruction of NIMA and attenuation of its kinase activity (Pu \& Osmani, 1995). It has recently been shown that NIMA is required for nuclear localization of the $\mathrm{p} 34^{\text {nimX }}-\mathrm{p} 56^{\text {nimE }} \mathrm{cdk}$ complex (Wu et al., 1998).

The experiments described in this paper were designed to determine if spore polarization is coordinated with nuclear division, and if so, to provide initial insight into the nature of the underlying regulatory mechanism(s). By examining the kinetics of spore polarization in a battery of strains possessing defined defects in nuclear division, the following conclusions were reached: (i) spore polarization is coupled to cell cycle progression, and (ii) the mitosis-promoting kinase NIMA may play a role in inhibiting spore polarization. In addition, evidence is presented that suggests that the link between spore polarization and nuclear division can be uncoupled by growth signals. The data supporting these conclusions and a tentative model for the temporal regulation of spore polarization are described below.

\section{METHODS}

Strains, genetic manipulations and growth conditions. All strains used in this study are described in Table 1 . Media used were YGV (2\% glucose, $0.5 \%$ yeast extract, $0.1 \%$ vitamin mix), MNV ( $1 \%$ glucose, $0.1 \%$ vitamin mix, nitrate salts, trace elements, $\mathrm{pH} \mathrm{6.5)} \mathrm{and} \mathrm{MNV} \mathrm{+} \mathrm{thr} \mathrm{(same} \mathrm{as} \mathrm{MNV,} \mathrm{except}$ $100 \mathrm{mM}$ L-threonine substituted for glucose). Vitamin mix, trace elements and nitrate salts were made as described by Kafer (1977). For all experiments, Sarstedt tubes containing YGV were inoculated with conidia at a density of $2.5 \times 10^{4}-$ $1 \times 10^{5} \mathrm{ml}^{-1}$ and gently poured into Petri dishes containing glass coverslips. The conidia settled to the bottom of the Petri dish and adhered tightly to the coverslips. At the appropriate times, coverslips with adherent cells were removed and processed for microscopy. All experiments were repeated at least twice.

For the measurement of polarization kinetics, conidia were incubated in $\mathrm{YGV}$ at either $43.5^{\circ} \mathrm{C}$ [for strains containing temperature-sensitive (ts) mutations] or $37^{\circ} \mathrm{C}$ (for non-ts strains) for $6 \mathrm{~h}$ to allow breaking of dormancy. Samples were then taken at hourly intervals up to $12 \mathrm{~h}$. For the experiments in which chemicals were used to block cell cycle progression, inhibitors were added at the time of inoculation into YGV, and conidia were incubated in their presence for $5 \mathrm{~h}$. Hydroxyurea (HU, Sigma) was used at a final concentration of $50 \mathrm{mM}$ (from a $2 \mathrm{M}$ stock solution in sterile water), and benomyl (a gift from Dupont) was used at a final concentration of $5 \mu \mathrm{g} \mathrm{ml} \mathrm{m}^{-1}$ (from a $500 \mu \mathrm{g} \mathrm{ml} \mathrm{m}^{-1}$ stock solution in $70 \%$ ethanol). Hyphae were released from cell cycle arrest by two sequential washes in pre-warmed YGV, followed by transfer to a new Petri dish containing fresh pre-warmed YGV. Following release, samples were taken at $30 \mathrm{~min}$ intervals over the next $3 \mathrm{~h}$.

Induction of the alcA promoter was accomplished by providing the relevant strains with $100 \mathrm{mM}$ threonine as sole carbon source (Waring et al., 1989). For these experiments, strains were inoculated into $\mathrm{YGV}$ and incubated at $37^{\circ} \mathrm{C}$ for $3 \mathrm{~h}$ to hasten the breaking of dormancy (conidia inoculated directly into $\mathrm{MNV}+$ thr take $>6 \mathrm{~h}$ to germinate). During this period of incubation, conidia underwent isotropic swelling but did not polarize. Coverslips were then washed twice with prewarmed $\mathrm{MNV}+\mathrm{thr}$, and were subsequently transferred to a Petri dish containing fresh prewarmed MNV + thr at $37^{\circ} \mathrm{C}$. Control (alcA uninduced) coverslips were kept in YGV at $37^{\circ} \mathrm{C}$. Samples were taken at $2 \mathrm{~h}$ intervals following the media shift.

For the nutritional shift, wild-type (A28) conidia were incubated at $37^{\circ} \mathrm{C}$ in YGV (rich media) for $2.5 \mathrm{~h}$. One set of coverslips were transferred to a Petri dish containing prewarmed YGV (rich media) and the incubation continued at $37^{\circ} \mathrm{C}$. The remaining coverslips were washed twice in prewarmed MNV (poor media), and then transferred into a Petri dish containing fresh pre-warmed MNV at $37^{\circ} \mathrm{C}$. Samples were taken at $30 \mathrm{~min}$ intervals following the nutritional shift. For some experiments, nocodazole (Sigma) was added to rich or poor media at a final concentration of $5 \mu \mathrm{g} \mathrm{m}^{-1}$ (from a stock solution of $5 \mathrm{mg} \mathrm{ml}^{-1}$ in DMSO).

Staining and microscopy. Preparation of samples, staining with Calcofluor and Hoechst 33258, and mounting of stained coverslips on glass slides were performed as previously 
Table 1. A. nidulans strains

\begin{tabular}{|c|c|c|}
\hline Strain & Genotype & Source* \\
\hline ASH486 & $w A 2$ & Laboratory stock \\
\hline ASH487 & $\operatorname{nim} A 5 ; w A 2$ & Laboratory stock \\
\hline A28 & pabaA6; biA1 & 1 \\
\hline A781 & $\operatorname{nim} A 5 ; w A 2$ & 1 \\
\hline SO75 & nimE6; riboA1; yA2 & 2 \\
\hline JD139† & nimL15; chaA1 & 3 \\
\hline ts 826 & $\begin{array}{l}\operatorname{nimN17;} \text { adE20, biA1; wA2, cnxE16; sC12; } \\
\text { meth } 1 \text {; nicA2; lacA1; choA1; chaA1 }\end{array}$ & 4 \\
\hline SWJ238 & nim $\mathrm{O} 18 ;$ riboA $1 ; y A 2$ & 5 \\
\hline SWJ280 & nimP22; pyroA4; chaA1 & 5 \\
\hline SWJ186 & nimQ20; choA1 & 5 \\
\hline SWJ310 & $\operatorname{nimR} 21 ;$ pabaA1 & 5 \\
\hline SO53 & $\operatorname{nim} T 23 ; w A 2$ & 2 \\
\hline SO65 & $\operatorname{nimX} 3(\mathrm{Y} 306 \mathrm{H}) ; w A 3 ;$ pyroA4; riboA1; yA2 & 2 \\
\hline PM131 & $\operatorname{bim} A 1 ;$ riboA $1 ; y A 2$ & 6 \\
\hline A777 & $\begin{array}{l}\text { bimC4, lacA1; adE20, biA1; wA2, cnxE16; sC12; } \\
\text { meth } 1 \text {; nicA2; choA1; chaA1 }\end{array}$ & 1 \\
\hline SA253† & hfaB3; yA2 & 7 \\
\hline SWJ010 & bimE7; pabaA1, yA2 & 6 \\
\hline PL11-2‡ & $\begin{array}{l}2 \times(\operatorname{alcA}: \operatorname{nim} A, \operatorname{nim} A 5 ; w A 2 ; \text { pyrG89, yA2; } \\
\text { cnxE16; choA1; chaA1 })\end{array}$ & 8 \\
\hline PL11-4‡ & $\begin{array}{l}4 \times(\text { alcA::nimA, nimA5; wA2; pyrG89, yA2; } \\
\text { cnxE16; choA1; chaA1) }\end{array}$ & 8 \\
\hline PL11-7‡ & $\begin{array}{l}7 \times(\operatorname{alc} A: \operatorname{nim} A, \operatorname{nim} A 5 ; w A 2 ; \text { pyrG89, yA2; } \\
\text { cnxE16; choA1; chaA })\end{array}$ & 8 \\
\hline
\end{tabular}

*1, Fungal Genetic Stock Center, Dept of Microbiology, University of Kansas Medical Center, Kansas City, KS, USA ; 2, Stephen Osmani, Geisinger Clinic, Danville, PA, USA; 3, John Doonan, John Innes Centre, Norwich, UK; 4, Ron Morris, University of Medicine and Dentistry of New Jersey, Piscataway, NJ, USA; 5, Steven James, Gettysburg College, Gettysburg, PA, USA ; 6, Peter Mirabito, University of Kentucky, Lexington, KY, USA; 7. Susan Assinder, University of Wales, Bangor, UK; 8, Anthony Means, Duke University Medical Center, Durham, NC, USA.

† May contain other uncharacterized markers.

$\ddagger$ For details of strain construction, see Lu \& Means (1994).

described (Harris et al., 1994). Slides were observed using an Olympus BMAX fluorescent microscope and DPLANAPO $\times 40$ and $\times 100$ (oil immersion) objectives. Spore size was measured using a calibrated eyepiece micrometer.

Measurements. For each time point, 200 spores were counted to determine the fraction that had polarized. Currently, the most convenient marker for spore polarization is the presence of a germ tube. Although recent observations suggest that polarization of the actin cytoskeleton occurs prior to germ tube emergence (Harris et al., 1999), this event cannot be reliably detected under the growth conditions used for the experiments described here (unpublished results). Thus, a spore was counted as polarized if it possessed a germ tube. Since conidia are spherical in shape, recently emerged germ tubes were readily detectable as small protruberances on the spore surface.

Levels of NIMA activity in different strains were indirectly assessed by determining the chromosome mitotic index (CMI). This is a fairly reliable indicator since activated NIMA kinase is known to trigger chromosome condensation in A. nidulans and other organisms (Osmani et al., 1991; O'Connell et al., 1994). Thus, for some of the experiments described here, the fraction of spores $(n=200)$ possessing highly condensed nuclei was determined at each time point. As an example, the level of chromosome condensation ( $22 \%$ vs $65 \%$ ) observed in strains PL11-4 (four integrated copies of alcA::nimA) and PL11-7 (seven integrated copies of alcA::nimA) $10 \mathrm{~h}$ after $\operatorname{alcA}(\mathrm{p})$ induction is directly proportional to nim $A$ copy number.

All experiments reported here were repeated at least twice with essentially indentical results. In all cases, representative results from one experiment are shown. Although the overall kinetics of spore polarization displayed some variability, there was always a consistent relationship between the different mutants and wild-type (e.g. nimA5 mutants always polarized 15-30 min earlier than wild-type).

\section{RESULTS}

\section{Spore polarization is delayed until the completion of the first mitotic division}

Germinating A. nidulans conidia establish a polarized axis of growth following an initial period of isotropic swelling. At the same time, the single nucleus present in the spore re-enters the cell cycle and undergoes a series 


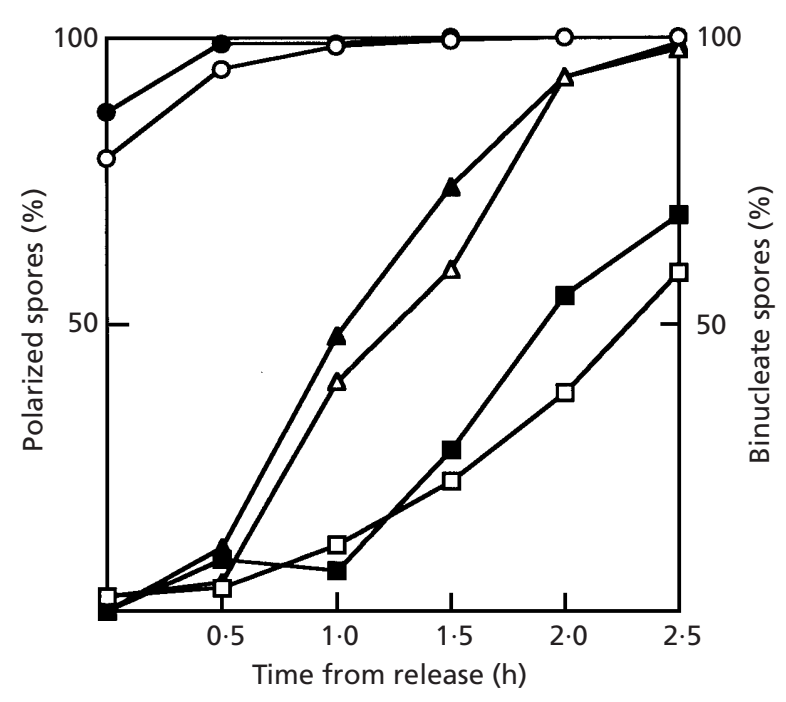

Fig. 1. Spore polarization requires completion of the first mitosis. Wild-type (A28) conidia were germinated on coverslips at $37^{\circ} \mathrm{C}$ in YGV containing $50 \mathrm{mM} \mathrm{HU}$ or $5 \mu \mathrm{g}$ benomyl ml $\mathrm{m}^{-1}$. Spores were released into drug-free media and monitored at 30 min intervals for polarization (open symbols) and nuclear division (filled symbols). Circles, untreated spores; triangles, spores treated with benomyl; squares, spores treated with HU.

of mitotic divisions unaccompanied by cytokinesis (Harris, 1997). These processes ultimately lead to the formation of a polarized hyphal cell that is multinucleate. However, it has not been determined if the morphogenetic events involved in spore polarization occur in a manner that is entirely independent of cell cycle progression, or if the two processes are in some way functionally linked with one another. To address this question, the progression of spore polarization relative to nuclear division was monitored in a wild-type strain. No spore possessing a single nucleus was found to be polarized at any time point. In contrast, a significant fraction of the spores containing two nuclei were polarized $(24 \%$ at $5 \mathrm{~h}, 36 \%$ at $6 \mathrm{~h})$, as were the majority of those which possessed four nuclei $(51 \%$ at 5 $\mathrm{h}, 87 \%$ at $6 \mathrm{~h}$ ). These results show that spore polarization is preceded by at least one round of mitosis.

The possibility that completion of the first mitotic division is a prerequisite for spore polarization was assessed by performing block and release experiments with inhibitors of cell cycle progression. Spores from the wild-type strain A28 were incubated in the presence of either HU or benomyl for $5 \mathrm{~h}$, followed by release into inhibitor-free media. Both drugs were found to significantly impair spore polarization (Fig. 1). Following $5 \mathrm{~h}$ in the presence of either HU or benomyl, no spore had undergone nuclear division and only $2.5 \%$ were polarized. In contrast, at the same time point, $87 \%$ of untreated spores were binucleate and a total of $79 \%$ had polarized. Furthermore, the degree of inhibition of spore polarization caused by HU was found to be dosedependent, as lower doses caused only a brief delay in polarization (unpublished results). These results suggest that the inability to complete the first mitotic division prevents spore polarization.

Following release from either the benomyl- or HUinduced blocks, the kinetics of spore polarization closely paralleled that of nuclear division (Fig. 1). For example, $1 \mathrm{~h}$ after release from the benomyl block, $48 \%$ of all spores were binucleate and $40 \%$ had polarized. Similarly, $2 \mathrm{~h}$ after release from the HU block, $55 \%$ of all spores were binucleate and $38 \%$ had polarized. The longer period of time required for spore polarization and nuclear division following release from the $\mathrm{HU}$ block is presumably because these cells were recovering from an S-phase arrest (Bergen \& Morris, 1983), whereas the benomyl-treated spores were recovering from arrest in mitosis (Orr \& Rosenberger, 1976). The tight coupling of spore polarization to nuclear division in these block and release experiments suggests that spore polarization is dependent upon completion of the first mitotic division. However, this dependency does not appear to be absolute as a small fraction $(<1 \%)$ of the spores that polarized upon release from the HU block were found to be uninucleate.

\section{Spore polarization in ts cell cycle mutants}

In A. nidulans, a large collection of ts mutants defective in cell cycle progression have been identified and characterized in detail (Morris, 1976; Doonan, 1992; Morris \& Enos, 1992; James et al., 1995). Although these mutants fail to complete the first mitotic division when conidia are incubated directly at restrictive temperature, most of them do undergo spore polarization and form an elongating hypha. However, it is not known if these mutations cause delays in spore polarization similar to those induced by treatment of wild-type spores with HU or benomyl. To address this question, the timing of spore polarization was examined in individual mutants incubated at restrictive temperature and compared to that of a wild-type strain. As described below, these mutations fell into three distinct categories based upon their effects on spore polarization (Table 2).

The largest group of mutations caused severe delays in spore polarization (Table 2), ranging from $\sim 1 \mathrm{~h}$ (nimE6) to $>6 \mathrm{~h}($ nimR21 and bimA1). These mutations affect a number of different stages of the cell cycle (Doonan, 1992; James et al., 1995), including (i) initiation of DNA replication (nimQ20, nimR21, nim O18), (ii) progression through $S$ phase (nimP22), (iii) regulation of mitosis (nimE6, bimA1) and (iv) spindle assembly (bimC4). Although these mutations prevent the completion of the first mitotic division in spores germinating at restrictive temperature (Table 2), spore polarization ultimately did occur. However, plating experiments revealed that spores possessing these mutations suffer from increased levels of aneuploidy and begin to lose viability when incubated at restrictive temperature for periods longer than $8 \mathrm{~h}$ (unpublished 
Table 2. Percentage spore polarization in ts cell cycle mutants

Spores were incubated in YGV for $12 \mathrm{~h}$ at $43.5^{\circ} \mathrm{C}$. The percentage of spores that had polarized was determined by DIC microscopy $(n=200)$.

\begin{tabular}{|c|c|c|c|c|}
\hline \multirow[t]{2}{*}{ Mutation (defect) } & \multicolumn{4}{|c|}{ Length of incubation $(h)$} \\
\hline & 6 & 7 & 8 & $12 \%$ \\
\hline Wild-type & $95 \cdot 5$ & $97 \cdot 5$ & 98 & ND \\
\hline $\operatorname{nim} A 5(\mathrm{G} 2 / \mathrm{M})$ & $98 \cdot 5$ & $94 \cdot 5$ & $93 \cdot 5$ & ND \\
\hline hfaB3 $(\mathrm{G} 2 / \mathrm{M})$ & $93 \cdot 5$ & 97 & 95 & $\mathrm{ND}$ \\
\hline $\operatorname{nim} T 23(\mathrm{G} 2 / \mathrm{M})$ & 90 & 99 & $98 \cdot 5$ & ND \\
\hline $\operatorname{nim} X 3(\mathrm{G} 1)$ & $98 \cdot 5$ & $97 \cdot 5$ & 99 & ND \\
\hline $\operatorname{nim} Q 20(\mathrm{G} 1 / \mathrm{S})$ & 23 & $68 \cdot 5$ & $79 \cdot 5$ & 92 \\
\hline $\operatorname{nimR} 21(\mathrm{G} 1 / \mathrm{S})$ & 6 & $23 \cdot 5$ & $49 \cdot 5$ & 54 \\
\hline $\operatorname{nim} \mathrm{O} 18(\mathrm{G} 1 / \mathrm{S})$ & 33 & 48 & $66 \cdot 5$ & 95 \\
\hline $\operatorname{nimP22}(\mathrm{S})$ & 16 & 57 & 76 & $92 \cdot 5$ \\
\hline nimE6 (G2/M) & $24 \cdot 5$ & $33 \cdot 5$ & 95 & $98 \cdot 5$ \\
\hline $\operatorname{bim} A 1(\mathrm{G} 2 / \mathrm{M})$ & 0 & 13 & $25 \cdot 5$ & 71 \\
\hline $\operatorname{bim} \mathrm{C} 4(\mathrm{M})$ & $23 \cdot 5$ & 52 & 80 & 96 \\
\hline $\operatorname{nimL15}(\mathrm{S})$ & $0 \cdot 5$ & $1 \cdot 5$ & $8 \cdot 5$ & $20 \cdot 5$ \\
\hline $\operatorname{nim} N 17(\mathrm{~S})$ & 2 & 1 & 16 & 26 \\
\hline $\operatorname{bimE7}(\mathrm{G} 2 / \mathrm{M})$ & $7 \cdot 5$ & 11 & 13 & 14 \\
\hline
\end{tabular}

ND, Not determined.

* The percentage of cells that completed mitosis following $12 \mathrm{~h}$ at $43.5^{\circ} \mathrm{C}$ were $(n=100): \operatorname{nimA5}, 5 ; \mathrm{hfaB3}, 73 ; \operatorname{nimT23}, 96$; nimQ20, 5; $\operatorname{nimR21,9;} \operatorname{nimO18,4;nimP22,~24;nimX3,~7;~}$ nimE6, ND; $\operatorname{bimA1}, 20 ; \operatorname{bim} C 4,7 ; \operatorname{nimL15}, 1 ; \operatorname{nimN} 17, \mathrm{ND}$; and bimE7, 3.

results). Thus, although the failure to properly complete replication or assemble a functional mitotic spindle appears to delay spore polarization by preventing the first mitotic division, these spores eventually do polarize at a time coincident with the onset of aneuploidy and lethality.

The second group of mutations essentially prevented spore polarization (Table 2). Even after $24 \mathrm{~h}$ incubation at restrictive temperature, fewer than $30 \%$ of the spores possessing these mutations were polarized. In each mutant, the spores that did polarize were uninucleate (Table 2) and polarization occurred at a time corresponding to the onset of aneuploidy and lethality (unpublished results). One of the mutations in this group (bimE7) affects the regulation of mitosis and causes spores to arrest with an elevated CMI $(\sim 75 \%$; Osmani et al., 1988). The other two mutations (nimL15 and nimN17) appear to cause arrest in interphase by preventing the completion of replication (Doonan, 1992).

The third group of mutations had no apparent effect on the timing of spore polarization (Table 2). Three of the four mutations in this group (nimA5, hfaB3, nim T23) affect the regulation of mitosis (Doonan, 1992; Morris \& Enos, 1992), whereas the fourth $(\operatorname{nimX} 3)$ causes arrest at START in late G1 (Osmani et al., 1994). Despite being unable to complete the first mitotic division (Table 2), nim $A 5$ and nim $X 3$ mutants exhibited polarization kinetics similar to those of a wild-type strain. In principle, the failure of the nim T23 and $h f a B 3$ mutations to delay spore polarization could be attributed to their inherent leakiness (e.g. by $12 \mathrm{~h}, 96 \%$ of nim T23 spores and $73 \%$ of $h f a B 3$ spores were binucleate; Table 2 ). However, although $90 \%$ of the spores from a nim 223 strain had polarized following $6 \mathrm{~h}$ incubation at restrictive temperature, only $34 \%$ had undergone nuclear division. Thus, these mutations appear to relieve the dependency of spore polarization on the completion of the first mitotic division.

\section{The NIMA kinase may link spore polarization to the completion of mitosis}

A possible mechanism by which spore polarization could be coupled to the completion of the first mitotic division was revealed by the analysis of the ts cell cycle mutants. Specifically, a mutation which prevents the accumulation of active NIMA kinase (nimA5; Ye $e$ al., 1995) permits spore polarization to occur without delay in the absence of mitosis. In contrast, a mutation which leads to abnormally high levels of NIMA kinase activity (bimE7; Ye et al., 1996) prevents polarization. Furthermore, polarization of wild-type spores is prevented by treatment with benomyl, which leads to a mitotic arrest with high levels of NIMA kinase activity (Osmani et al., 1991). The inhibition of spore polarization does not appear to be due to defective mitosis per se since bimA1 and bim C4 mutants are able to polarize despite undergoing aberrant mitosis. These results suggest that the inhibition of spore polarization may in some manner be due to the effects of NIMA kinase activity. The following two experiments were performed to further test this idea.

If the increase in NIMA kinase activity which accompanies progression through G2 prevents spores from polarizing, then it might be expected that the loss of kinase activity in nimA mutants would lead to premature polarization. Consistent with this notion, spores from a nimA5 strain were observed to consistently undergo polarization 15-30 min earlier than those from an isogenic wild-type strain (Fig. 2). Moreover, the volume of nimA5 spores at the time of polarization was less than that of wild-type spores $\left(43 \cdot 8 \pm 15 \cdot 7\right.$ vs $\left.62 \cdot 6 \pm 20 \cdot 3 \mu \mathrm{m}^{3}\right)$. This result suggests that NIMA kinase activity may play a role in delaying spore polarization until mitosis is complete.

If NIMA kinase activity has a direct role in the inhibition of spore polarization, then artificial induction of kinase activity might be expected to prevent polarization in a dose-dependent manner. This notion was tested by monitoring spore polarization in a set of strains containing increasing doses of the $\operatorname{nim} A$ gene expressed under the control of the inducible alcA promoter ( $\mathrm{Lu} \&$ Means, 1994). The strains PL11-2, PL11-4 and PL11-7 


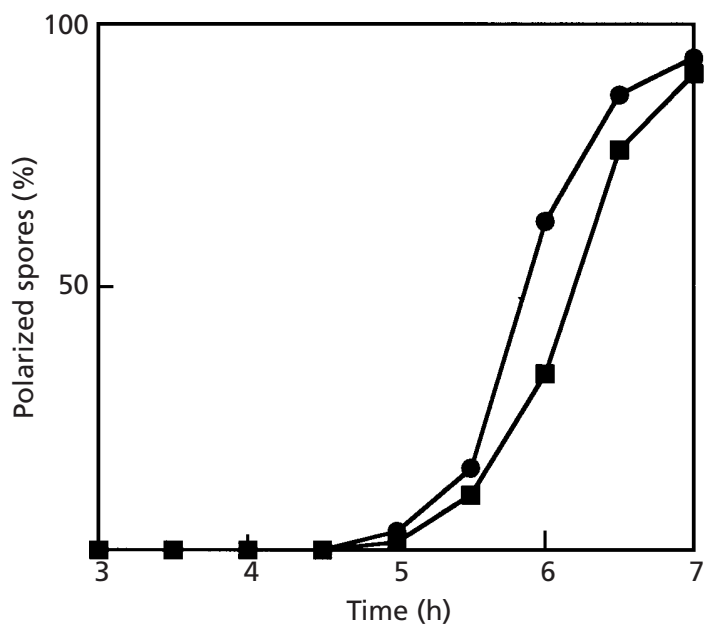

Fig. 2. The timing of spore polarization is advanced in nimA5 mutants. Conidia from a wild-type strain (ASH486; squares) and a nimA5 strain (ASH487; circles) were germinated on coverslips in YGV at $43.5^{\circ} \mathrm{C}$ for $7 \mathrm{~h}$. Spore polarization $(n=200)$ was monitored at 30 min intervals.

possess two, four and seven integrated copies of alcA::nimA, respectively (Lu \& Means, 1994). Spores from these strains and from a wild-type control were initially incubated on rich glucose media to hasten the breaking of dormancy. Under these conditions, the alc $A$ promoter is shut off and $\operatorname{nim} A$ is not expressed (Lu \& Means, 1994). The spores were subsequently transferred to inducing media while they were undergoing isotropic swelling. This lead to the rapid induction of nimA expression and the accumulation of NIMA kinase activity, as indicated by the dose-dependent increase in the CMI (i.e. at $2 \mathrm{~h}$ post-induction, the CMI was 2, 5, 27 and $74 \%$, respectively, in the wild-type control strain, PL11-2, PL11-4 and PL11-7). Increased levels of NIMA kinase were found to inhibit spore polarization in a dose-dependent manner (Fig. 3). For example, at $10 \mathrm{~h}$ post-induction, when all wild-type spores had undergone nuclear division and $97 \%$ had polarized, $34 \%$ of PL11-4 spores were binucleate and only $35 \%$ had polarized. Similarly, only $6 \%$ of PL11-7 spores were binucleate and only $9 \%$ had polarized at this time. Although these observations may reflect general effects of excess NIMA on chromosome condensation and gene expression, they do hint at a possible relationship between NIMA kinase activity and the inhibition of spore polarization.

\section{An additional mechanism inhibits polarization during S phase}

The results described above suggest that spore polarization is coupled to cell cycle progression by the activity of the NIMA kinase. However, spore polarization in nimA5 mutants is still dependent upon progression through $S$ phase. In particular, when incu-

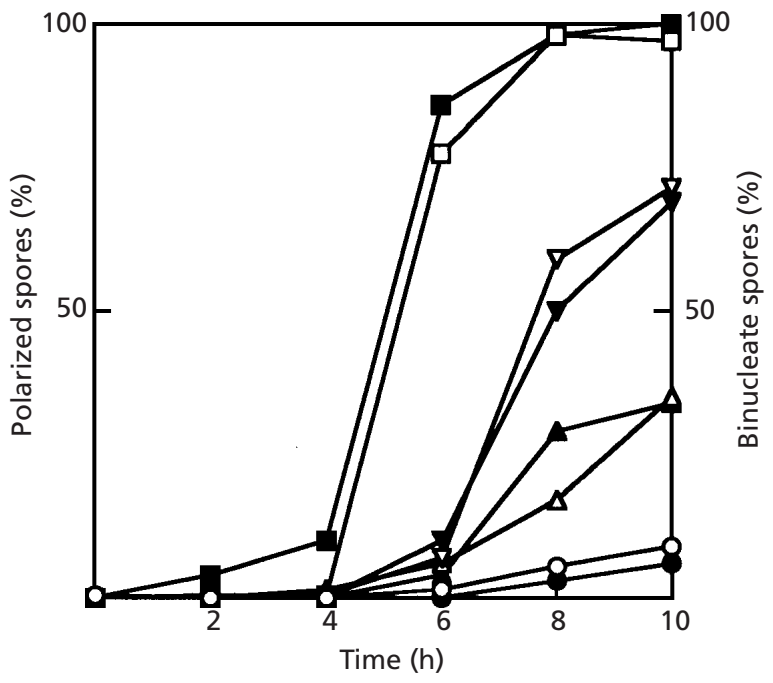

Fig. 3. Increased levels of NIMA block spore polarization. Conidia from a wild-type strain (A28; squares), or from strains possessing two copies (PL11-2; inverted triangles), four copies (PL11-4; triangles), or seven copies (PL11-7; circles) of alcA::nimA were germinated on coverslips in YGV at $37{ }^{\circ} \mathrm{C}$ for $3 \mathrm{~h}$. Coverslips were washed and transferred to $M N V+$ thr medium to induce expression from the alcA promoter. Spore polarization ( $n=200$; open symbols) and nuclear division ( $n=200$; filled symbols) were monitored at $2 \mathrm{~h}$ intervals following transfer.

bated at $43.5^{\circ} \mathrm{C}$ in the presence of $50 \mathrm{mM} \mathrm{HU}$, polarization was blocked just as effectively in nimA5 mutants as it was in wild-type controls (i.e. in both populations, only $5 \%$ of spores polarized; $n=200)$. This observation implies that a NIMA-independent mechanism functions to prevent polarization as germinating spores progress through their first $S$ phase. The existence of such a mechanism is not altogether surprising since NIMA kinase activity is reported to be extremely low during S phase (Osmani et al., 1991).

\section{The link between spore polarization and mitosis can be uncoupled by growth signals}

The experiments described above demonstrate that spore polarization is coupled to the completion of the first mitosis when spores are incubated in rich media (i.e. YGV). To determine if the timing of spore polarization relative to nuclear division could be modulated by growth signals, the kinetics of spore polarization and nuclear division were monitored in germinating spores subjected to a nutritional shift. Transfer of germinating spores from rich to poor media (YGV to MNV; Fig. 4) caused an apparent slowing of cell cycle progression. For example, the percentage of binucleate spores at $2 \mathrm{~h}$ post-shift was dramatically lower in the population shifted to MNV compared to a control population shifted to YGV (i.e. 23 vs $77 \%$; Fig. 4a). Despite the delay in nuclear division, spore polarization was accelerated by the shift to a poorer media source. 


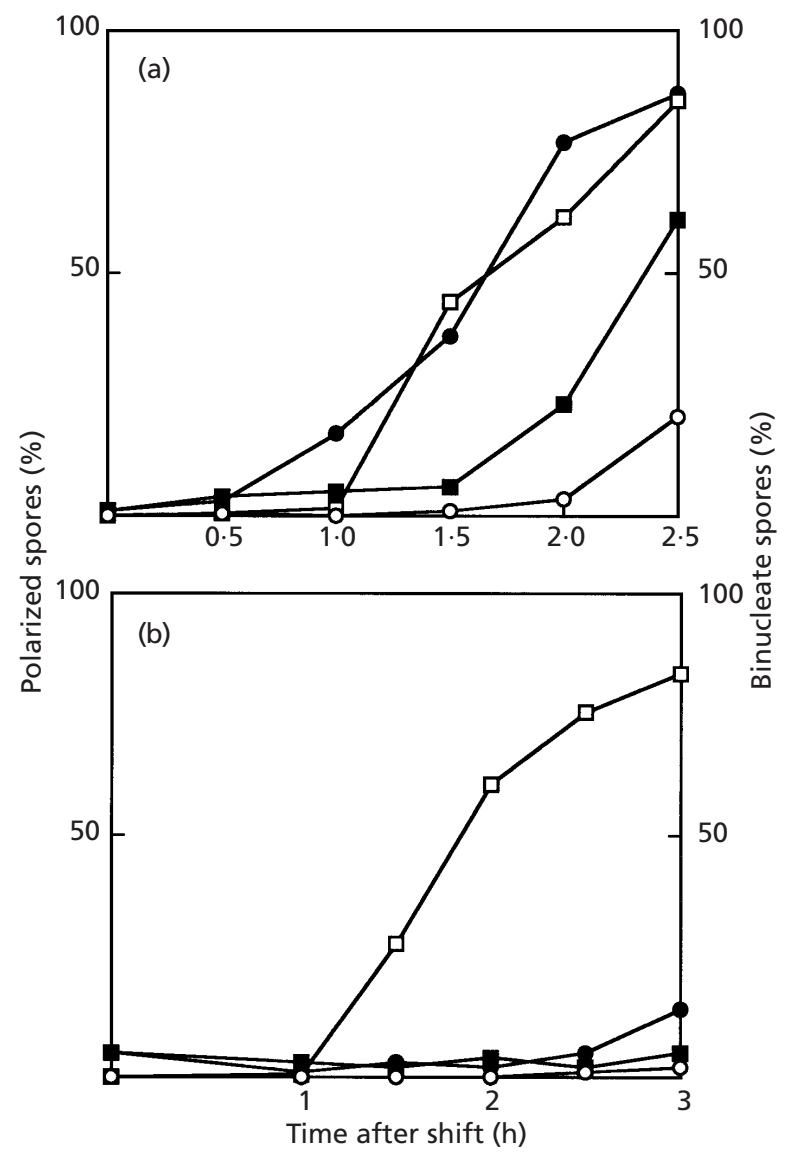

Fig. 4. Growth signals can uncouple the link between spore polarization and nuclear division. Wild-type (A28) conidia were germinated on coverslips at $37{ }^{\circ} \mathrm{C}$ in YGV for $2.5 \mathrm{~h}$. Coverslips were washed and transferred to (a) YGV (rich media) or MNV (poor media), or (b) YGV or MNV each containing $5 \mu \mathrm{g}$ nocodazole $\mathrm{ml}^{-1}$. Spore polarization ( $n=200$; open symbols) and nuclear division ( $n=200$; filled symbols) were monitored at 30 min intervals following the shift. Circles, YGV; squares, MNV.

Whereas only $3.5 \%$ of the spores in the control population had polarized by $2 \mathrm{~h}$ post-shift, $61.5 \%$ of the spores shifted to MNV had polarized by this time (Fig. $4 a)$. Furthermore, since accelerated polarization occurred even when spores were shifted to poor media containing nocodazole (Fig. 4b), the shift-induced stimulation of spore polarization did not require nuclear division. These observations support the notion that growth signals can overrule the effect of cell cycle signals on spore polarization.

\section{DISCUSSION}

It has become increasingly apparent that in yeasts complex regulatory networks function to coordinate polarized morphogenesis with both the nuclear division cycle and cellular growth (Kron \& Gow, 1995). The experiments described here were designed to test the idea that similar networks may operate during conidio- spore germination in the filamentous fungus A. nidulans. Since these uninucleate spores give rise to multinucleate hyphal cells, morphogenesis could conceivably proceed in a manner that is entirely independent of nuclear division. Instead, the results presented here suggest that these processes are linked. Specifically, it is shown that: (i) under optimal growth conditions, spore polarization is coupled to completion of the first mitosis; (ii) the inhibition of spore polarization during mitosis may be dependent upon NIMA kinase activity; and (iii) growth signals can uncouple the link between spore polarization and nuclear division.

\section{Spore polarization is coupled to completion of the first mitosis}

Conidiospores germinating on rich media (i.e. YGV) undergo a round of nuclear division prior to the emergence of a germ tube. Two independent lines of evidence demonstrate that completion of this intraconidial mitosis is a prerequisite for spore polarization. First, polarization does not occur if cell cycle progression is blocked by the inhibitors HU or benomyl. In addition, as would be expected if polarization depends upon the completion of mitosis, spores recovering from a mitotic block (benomyl; Orr \& Rosenberger, 1976) polarized much more rapidly than spores recovering from an Sphase block (HU; Bergen \& Morris, 1983). Second, spore polarization was delayed by a number of different ts mutations that block cell cycle progression during interphase (nimE6, nim O18, nimP22, nimQ20 and nimR21) or mitosis (bimA1 and bimC4). The extent of the polarization delay depended upon the particular mutation, though it typically ranged from 1 to $6 \mathrm{~h}$. The delay is presumably not caused by an indirect effect on growth since, for example, HU has no effect on RNA or protein synthesis in A. nidulans (Bergen \& Morris, 1983). Instead, the most likely explanation for the delay is failure to reach a specific point in the nuclear division cycle (i.e. an event associated with the completion of mitosis) at which the spore commits to polarization. The observation that the mutant spores ultimately did polarize despite the failure to complete mitosis is critical since it demonstrates that these mutations do not prevent morphogenesis per se. It also suggests that the normal coupling of polarization to completion of mitosis can be overidden by other regulatory responses (i.e. stress responses).

\section{The NIMA kinase may play a role in coupling spore polarization to mitosis}

Analysis of the kinetics of spore polarization in the ts cell cycle mutants provided insight into a mechanism that could conceivably couple polarized morphogenesis to nuclear division. Specifically, polarization was essentially inhibited in a mutant that accumulates excess amounts of fully active, hyperphosphorylated NIMA kinase (i.e bimE7; Ye et al., 1996). Similarly, polarization was severely delayed in another mutant 
that displays higher than normal NIMA kinase activity (bimA1; Lies et al., 1998). Since BIMA and BIME are components of the anaphase-promoting complex (Peters et al., 1996; Lies et al., 1998), which is required for the targeted ubiquitination of cell cycle regulators (Townsley \& Ruderman, 1998), activated NIMA may accumulate in these mutants because it cannot be properly degraded. In contrast, polarization proceeded without delay in mutants that fail to accumulate hyperphosphorylated NIMA (i.e. nimA5, nim T23; Ye et al., 1995). Indeed, polarization was slightly advanced relative to wild-type when nimA5 mutants were examined in detail. These results suggest that the inhibition of polarized morphogenesis is associated with the presence of the fully active, hyperphosphorylated form of NIMA. Two additional observations provide support for this idea. First, increased expression of the nim $A$ gene leads to a dose-dependent inhibition of spore polarization, even under conditions in which nim $A$ mutations do not normally affect polarization (i.e. minimal media). Second, expression of a truncated form of NIMA that can be hyperphosphorylated, but not degraded (Pu \& Osmani, 1995), prevents polarization (unpublished results). The simplest interpretation of these results is that activated NIMA kinase may inhibit spore polarization.

The potential role of NIMA in coupling spore polarization to the completion of mitosis can be explained by the following model. As spores pass through the G2/M boundary of the first mitosis, hyperphosphorylation of NIMA by $\mathrm{p} 34^{\text {nimX }}$ leads to attainment of full kinase activity (Ye et al., 1995). The activated kinase promotes events required for mitosis, and in addition inhibits spore polarization. Proteolytic degradation of NIMA triggers exit from the ensuing mitosis (Pu \& Osmani, 1995), and also permits spore polarization by removing the inhibitory signal. The ability of activated NIMA kinase to prevent spore polarization could be either a direct or indirect effect. For example, NIMA could directly phosphorylate, and hence inactivate, a substrate that is required for polarization. Alternatively, activated NIMA could indirectly inhibit polarization by promoting a mitotic event such as chromosome condensation (Osmani et al., 1991; O'Connell et al., 1994). In this scenario, expression of a gene required for morphogenesis is suppressed when chromosomes are in a condensed state. Distinguishing between these possibilities will not be straightforward, since a mutation or condition that uncouples NIMA activation from chromosome condensation does not yet exist.

\section{Additional mechanisms prevent spore polarization prior to mitosis}

Results presented here demonstrate that drugs (i.e. HU; Bergen \& Morris, 1983) or ts mutations (i.e. nimL15, nimN17; Doonan, 1992) that block progression through $S$ phase also inhibit spore polarization. Since NIMA levels are very low in S-phase cells (Osmani et al., 1991), this effect is presumably not attributable to NIMA kinase activity. In support of this notion, spore polarization is blocked in nimA5 mutants treated with HU, or in nimL15 nimA5 double mutants (unpublished results). How then is polarization prevented when spores are progressing through $S$ phase? Activation of the checkpoint pathway that responds to incompletely replicated DNA could conceivably inhibit polarization. However, the observation that polarization is still blocked by HU when the replication checkpoint is abrogated rules out this possibility (unpublished results). Alternatively, expression of a gene required for morphogenesis may be suppressed when chromosomes are in a replicative state. This idea could be tested by examining the expression pattern of genes required for polarized morphogenesis.

\section{Growth signals can uncouple spore polarization from nuclear division}

The coupling of polarization to the completion of mitosis in germinating conidiospores could reflect the activity of a size control mechanism that establishes a ratio of nuclei to cytoplasm that is optimal for subsequent hyphal growth (Trinci, 1978). If so, poorer growth conditions should then alter the size setting and thus influence the timing of spore polarization relative to nuclear division. Consistent with this notion, conidiospores germinating on MNV media polarize prior to the first mitosis and, under these conditions, polarization is not blocked when spores are trapped in mitosis with condensed chromosomes (unpublished results). Moreover, shifting germinating conidiospores from rich to poor media induces polarization in the absence of nuclear division. These observations suggest that conidiospores can rapidly sense growth conditions and adjust the size control accordingly. In addition, they show that the effects of the cell cycle on spore polarization can be overridden by growth signals.

\section{Why is spore polarization not delayed in $\operatorname{nim} X 3$ mutants?}

Under the conditions used in the experiments described here, the nim X3 mutation arrests cell cycle progression in G1 (Osmani et al., 1994). However, despite failing to proceed through the cell cycle to the completion of mitosis, spore polarization is not delayed by the nim X3 mutation. Furthermore, HU has no effect on the kinetics of spore polarization in nimX3 mutants (unpublished results). Why then, do these mutants undergo spore polarization despite not completing mitosis? One possible explanation is that growth signals can promote polarization in the absence of inhibitory cell cycle signals (i.e. generated when conidiospores are in $S$ phase or mitosis). In this model, although spores germinating in rich media are competent to polarize at START, cell cycle signals delay polarization until the completion of mitosis. This delay may ensure the optimal ratio of nuclei to cytoplasm for hyphal growth. In nimX3 mutants, or in wild-type spores germinating on poor 
media, the cell cycle signal is not generated and spores can thus polarize prior to the completion of mitosis.

\section{ACKNOWLEDGEMENTS}

I thank John Doonan, Steven James, Peter Mirabito, Tony Means, Ron Morris and Stephen Osmani for providing strains. I also thank Xiang Ye and Tom Wolkow for fruitful discussions.

This work was supported by a New Investigator Award from The Patrick and Catherine Weldon Donaghue Medical Research Foundation.

\section{REFERENCES}

Baroni, M. D., Monti, P. \& Alberghina, L. (1994). Repression of growth-regulated G1 cyclin expression by cAMP in budding yeast. Nature 371, 339-342.

Bergen, L. \& Morris, N. R. (1983). Kinetics of the nuclear division cycle of Aspergillus nidulans. J Bacteriol 156, 155-160.

Bruno, K. S., Aramayo, R., Minke, P. F., Metzenberg, R. L. \& Plamann, M. (1996). Loss of growth polarity and mislocalization of septa in a Neurospora mutant altered in the regulatory subunit of cAMP-dependent protein kinase. EMBO J 15, 5772-5782.

Dirick, L., Bohm, T. \& Nasmyth, K. (1995). Roles and regulation of Cln-Cdc28 kinases at the start of the cell cycle of Saccharomyces cerevisiae. EMBO J 14, 4803-4813.

Doonan, J. H. (1992). Cell division in Aspergillus. J Cell Sci 103, 599-611.

d'Enfert, C. (1997). Fungal spore germination: insights from the molecular genetics of Aspergillus nidulans and Neurospora crassa. Fungal Genet Biol 21, 163-172.

Gold, S., Duncan, G., Barrett, K. \& Kronstad, J. (1994). cAMP regulates morphogenesis in the fungal pathogen Ustilago maydis. Genes Dev 8, 2805-2816.

Gow, N. A. R. (1995). Tip growth and polarity. In The Growing Fungus, pp. 277-299. Edited by N. A. R. Gow \& G. M. Gadd. London: Chapman \& Hall.

Harris, S. D. (1997). The duplication cycle in Aspergillus nidulans. Fungal Genet Biol 22, 1-12.

Harris, S. D., Morrell, J. L. \& Hamer, J. E. (1994). Identification and characterization of Aspergillus nidulans mutants defective in cytokinesis. Genetics 136, 517-532.

Harris, S. D., Hofmann, A. F., Tedford, H. W. \& Lee, M. P. (1999). Identification and characterization of genes required for hyphal morphogenesis in the filamentous fungus Aspergillus nidulans. Genetics 151, 1015-1025.

James, S. W., Mirabito, P. M., Scacher, P. C. \& Morris, N. R. (1995). The Aspergillus nidulans bimE (blocked-in-mitosis) gene encodes multiple cell cycle functions involved in mitotic checkpoint control and mitosis. J Cell Sci 108, 3485-3499.

Kafer, E. (1977). Meiotic and mitotic recombination in Aspergillus and its chromosomal aberrations. Adv Genet 19, 33-131.

Kron, S. J. \& Gow, N. A. R. (1995). Budding yeast morphogenesis: signalling, cytoskeleton, and cell cycle. Curr Opin Cell Biol 7, 845-855.

Kronstad, J. (1998). Signalling via cAMP in fungi: interconnections with mitogen-activated protein kinase pathways. Arch Microbiol 170, 395-404.

Lew, D. J. \& Reed, S. I. (1993). Morphogenesis in the yeast cell cycle: regulation by Cdc28 and cyclins. J Cell Biol 120, 1305-1320.

Lies, C. M., Cheng, J., James, S. W., Morris, N. R., O'Connell, M. J.
\& Mirabito, P. M. (1998). BIMA ${ }^{\mathrm{APC} 3}$, a component of the Aspergillus anaphase promoting complex/cyclosome, is required for $a G_{2}$ checkpoint blocking entry into mitosis in the absence of NIMA function. J Cell Sci 111, 1453-1465.

Lu, K. P. \& Means, A. R. (1994). Expression of the noncatalytic domain of the NIMA kinase causes a $G_{2}$ arrest in Aspergillus nidulans. EMBO J 13, 2103-2113.

Madden, K., Sheu, Y.-J., Baetz, K., Andrews, B. \& Snyder, M. (1997). SBF cell cycle regulator as a target of the yeast PKC-MAP kinase pathway. Science 275, 1781-1784.

Morris, N. R. (1976). Mitotic mutants of Aspergillus nidulans. Genet Res 26, 237-254.

Morris, N. R. \& Enos, A.P. (1992). Mitotic gold in a mold: Aspergillus genetics and the biology of mitosis. Trends Genet $\mathbf{8}$, 32-37.

O'Connell, M. J., Osmani, A. H., Morris, N. R. \& Osmani, S. A. (1992). An extra copy of nim $E^{\text {cyclinB }}$ elevates pre-MPF levels and partially suppresses mutation of nim $T^{\mathrm{cdc} 25}$ in Aspergillus nidulans. EMBO J 11, 2139-2149.

O'Connell, M. J., Norbury, C. \& Nurse, P. (1994). Premature chromatin condensation upon accumulation of NIMA. EMBO J 13, 4926-4937.

Orr, E. \& Rosenberger, R. F. (1976). Determination of the execution points of mutations in the nuclear replication cycle of Aspergillus nidulans. J Bacteriol 126, 903-906.

Osmani, S.A. \& Ye, X.S. (1996). Cell cycle regulation in Aspergillus by two protein kinases. Biochem J 317, 633-641.

Osmani, S. A., Engle, D. B., Doonan, J. H. \& Morris, N. R. (1988). Spindle formation and chromatin condensation in cells blocked at interphase by mutation of a negative cell cycle control gene. Cell 52, 241-251.

Osmani, A. H., O’Donnell, K. O., Pu, R. T. \& Osmani, S. A. (1991). Activation of the $\operatorname{nim} A$ protein kinase plays a unique role during mitosis that cannot be bypassed by absence of the bimE checkpoint. EMBO J 10, 2669-2679.

Osmani, A. H., van Peij, N., Mischke, M., O’Connell, M. J. \& Osmani, S. A. (1994). A single p34 ${ }^{\mathrm{cdc} 2}$ protein kinase (encoded by nim $\left.\mathrm{X}^{\mathrm{cdc} 2}\right)$ is required at $\mathrm{G}_{1}$ and $\mathrm{G}_{2}$ in Aspergillus nidulans. J Cell Sci 107, 1519-1528.

Peters, J., King, R. W., Hoog, C. \& Kirschner, M. W. (1996). Identification of BIME as a subunit of the anaphase-promoting complex. Science 274, 1199-2001.

Pringle, J. R. \& Hartwell, L. H. (1981). The Saccharomyces cerevisiae cell cycle. In The Molecular Biology of the Yeast Saccharomyces: Life Cycle and Inheritance, pp. 97-142. Edited by J. N. Strathern, E. W. Jones \& J. R. Broach. Cold Spring Harbor, NY: Cold Spring Harbor Laboratory.

Pu, R. T. \& Osmani, S. A. (1995). Mitotic destruction of the cell cycle regulated NIMA protein kinase of Aspergillus nidulans is required for mitotic exit. EMBO J 14, 995-1003.

Som, T. \& Kolaparthi, V. S. R. (1994). Developmental decisions in Aspergillus nidulans are modulated by Ras activity. Mol Cell Biol 14, 5333-5348.

Tokiwa, G., Tyers, M., Volpe, T. \& Futcher, B. (1994). Inhibition of G1 cyclin activity by the Ras/cAMP pathway in yeast. Nature 371, 342-345.

Townsley, F. M. \& Ruderman, J. V. (1998). Proteolytic ratchets that control progression through mitosis. Trends Cell Biol 8, 238-244.

Trinci, A.P. J. (1978). The duplication cycle and vegetative development in moulds. In The Filamentous Fungi, vol. III, 
Developmental Mycology, pp. 132-163. Edited by J. E. Smith \& D. R. Berry. New York: Wiley.

Waring, R. B., May, G. S. \& Morris, N. R. (1989). Characterization of an inducible expression system in Aspergillus nidulans using alc $A$ and tubulin-encoding genes. Gene 79, 119-130.

Wu, L., Osmani, S. A. \& Mirabito, P. M. (1998). A role for NIMA in the nuclear localization of cyclin B in Aspergillus nidulans. J Cell Biol 141, 1575-1587.

Ye, X. S., Xu, G., Pu, R. T., Fincher, R. R., McGuire, S. L., Osmani, A. H. \& Osmani, S. A. (1995). The NIMA protein kinase is hyper- phosphorylated and activated downstream of $\mathrm{p} 34^{\mathrm{cdc} 2} /$ cyclin $\mathrm{B}$ : coordination of two mitosis promoting kinases. EMBO J 14, 986-994.

Ye, X. S., Fincher, R. R., Tang, A., O’Donnell, K. \& Osmani, S. A. (1996). Two S-phase checkpoint systems, one involving the function of both BIME and Tyr15 phosphorylation of $\mathrm{p} 34^{\mathrm{cdc} 2}$, inhibit NIMA and prevent premature mitosis. EMBO J 15, 3599-3610.

Received 16 March 1999; revised 17 June 1999; accepted 5 July 1999. 